\title{
MRI and DWI: the future in cervical cancer?
}

\author{
Daniel Grossi Marconi*, Erick Rauber, Paula de Cassia Soares \\ Department of Radiation Oncology, Barretos Cancer Hospital, Barretos/SP, Brazil
}

Received September 24, 2013; Revised September 26, 2013; Accepted September 27, 2013; Published Online September 27,2013

\section{Editorial}

Cervical cancer is the third leading cause of female cancer worldwide and is the second most common cause of cancer related deaths in women in undeveloped countries. ${ }^{1}$ The incident rate varies with the prevalence of risk factors and the lack of adequate screening programs, reaching 80 cases per 100,000 inhabitants in Recife, Brazil. ${ }^{2}$ Despite advances in treatment, cervical cancer still maintains high rates of morbidity and mortality - the recurrence rate and associated death is approximately $30 \%$. Data from the UK show that one third of patients will die within 5 years of diagnosis. ${ }^{3}$ For all these reasons cervical cancer can be considered a public health issue especially in developing countries.

The International Federation of Gynecology and Obstetrics (FIGO) stage system is the most widely used for cervical cancer. This system emphasizes the clinical parameters at the expense of morphological and functional examinations, which facilitates its applicability in developing countries where additional tests are expensive and not available. The main limitations are the fact that this system is examiner dependent, difficult to reproduce, and difficult to perform in obese patients or those with unfavorable anatomy.

Although without altering the classification proposed by FIGO, exams are valuable in this disease as they tend to guide the practitioner toward more accurate treatment. Furthermore, they are important to assess the response to treatment, which is of paramount importance since additional therapies (i.e. hysterectomy) may be used in cases of persistent disease.

The 18-FDG PET stands out as the most used and studied

${ }^{*}$ Corresponding author: Daniel Grossi Marconi, Department of Radiation Oncology, Barretos Cancer Hospital, Barretos/SP,

Brazil. Email: dgmarconi@gmail.com

Cite this article as:

Marconi D, Rauber E, Soares P. MRI and DWI: the future in

cervical cancer? Int J Cancer Ther Oncol 2013;1(1):01018.

DOI: $10.14319 /$ ijcto.0101.8 functional test in cervical cancer. Unfortunately, PET is not available to many treatment centers around the world, especially in those that have the highest incidence rates - as the funds needed to obtain its tracer make it cost prohibitive.

In this context, a much more cost effective relation is the MRI. Stenstedt et al. ${ }^{4}$ studied the impact of MRI in staging and follow-up of cervical lesions and concluded that the addition of this examination alter the staging proposed by FIGO and changes the treatment plan in many cases. In 2013, Kraljevic et al. ${ }^{5}$ performed a study comparing the FIGO staging and MRI preoperatively and correlated these findings with the pathological outcome in patients treated surgically. They concluded that MRI is better than clinical staging (accuracy of $90.9 \%$ versus $79.0 \%$ ). ${ }^{5}$

Novel advances such as diffusion weighted image (DWI) (sequence that is sensitive to the random motion of water molecules - i.e., Brownian motion) allow us to evaluate changes during therapy. Restriction to this diffusion movement is directly associated to the degree of cellularity of the tissue and thus is related to primary malignancy and metastasis. ${ }^{6}$ An actual issue is whether its changes are predictive of response: the DWI derived apparent diffusion coefficient (ADC) is capable to quantify the magnitude of this water diffusion and has been used extensively as a biomarker for therapeutic response in many cancer types.

Emerging studies seek to consolidate the actual role of MRI and their different weights in the staging and therapeutic monitoring of cervical cancer. Advantages such as cost-effectiveness (pathology is predominant in developing countries) and absence of need of contrast (essential in cases where there is impairment of renal function) add to this exam key features to this pathology. It is not difficult to predict that if new studies demonstrate in practice these advantages, MRI/DWI could be considered essential in the future. 


\section{References}

1. Ferlay J, Shin HR, Bray F, Forman D, Mathers C, Parkin DM. GLOBOCAN (2008) Cancer incidence and mortality worldwide. International Agency for Research on Cancer, Lyon. 2010; 127: 2893-2917. CrossRef

2. Jemal A, Siegal R, Xu J, Ward E. Cancer Statistics, 2010. CA Cancer J Clin 2010; 60: 277-300. CrossRef

3. Cancer Research UK (2010) Cervical cancer: survival statistics. Available via http://info.cancerresearchuk.org/cancerstats Accessed on September 21, 2013.

4. Stenstedt K, Hellström AC, Fridsten S, Blomqvist L. Impact of MRI in the management and staging of cancer of the uterine cervix. Acta Oncol 2011; 50:420-426. CrossRef
5. Kraljević Z, Visković K, Ledinsky M, Zadravec D, Grbavac I, Bilandzija M, Soljacić-Vranes H, Kuna K, Klasnić K, Krolo I. Primary uterine cervical cancer: correlation of preoperative magnetic resonance imaging and clinical staging (FIGO) with histopathology findings. Coll Antropol 2013; 37:561-568.

6. Malayeri AA, El Khouli RH, Zaheer A, Jacobs MA, Corona-Villalobos CP, Kamel IR, Macura KJ. Principles and applications of diffusion-weighted imaging in cancer detection, staging, and treatment follow up. Radiographics 2011; 31:1773-1791. CrossRef 